\title{
Treatment options in advanced renal cell carcinoma after first-line treatment with vascular endothelial growth factor receptor tyrosine kinase inhibitors
}

\author{
Naveen S. Basappa, MD, FRCPC \\ Department of Medical Oncology, Cross Cancer Institute, University of Alberta, Edmonton, AB, Canada
}

Cite as: Can Urol Assoc J 2016;10(11-12Supp17):S242-4.

http://dx.doi.org/10.5489/cuaj.4292

\section{Abstract}

Targeted therapy for metastatic renal cell carcinoma (mRCC) was introduced a decade ago and since then, a number of therapeutic options have been developed. Vascular endothelial growth factortargeted therapy is the widely accepted first-line option for mRCC. After progression, treatment in the second-line setting has typically been with either axitinib or everolimus. However, with the advent of several new agents demonstrating efficacy in the second-line setting, including nivolumab, cabozantinib, and the combination of lenvatinib and everolimus, the treatment paradigm has shifted toward these novel therapies with improved patient outcomes.

\section{Introduction}

The current approach to the treatment of metastatic renal cell carcinoma (mRCC) consists of sequential administration of single agents that target the vascular endothelial growth factor (VEGF) or mammalian target of rapamycin (mTOR) pathways. In Canada, a VEGF receptor-tyrosine kinase inhibitor (VEGFR-TKI) is the widely accepted treatment of choice in the first-line setting, namely sunitinib or pazopanib, for patients with mRCC. The mTOR inhibitor temsirolimus is a recognized option in select situations, ${ }^{1}$ although rarely used.

Following progression in the first-line setting, the VEGFRTKI axitinib or the mTOR inhibitor everolimus are the recommended second-line options in Canada; ${ }^{1}$ however, three new options with demonstrated efficacy in the second-line setting have arisen - nivolumab (a programmed death 1 [PD-1] inhibitor), cabozantinib (a TKI that inhibits VEGFR, $M E T$, and $A X L$ ), and the combination of lenvatinib (a VEGFR and fibroblast growth factor receptor [FGFR] TKI inhibitor) and everolimus.

\section{Second-line treatment options}

\section{Axitinib}

Axitinib is a highly selective inhibitor of VEGFR-1, -2, and $-3 .^{2}$ In an open-label, phase 2 study of 62 patients with sorafenib-refractory mRCC of any subtype, axitinib showed antitumour activity with mild-to-moderate toxicities. ${ }^{2}$ The randomized, phase 3 AXIS trial directly compared the efficacy and safety of axitinib with those of sorafenib in 723 patients with advanced RCC from 175 centres in 22 countries. ${ }^{3}$ Previous treatments included sunitinib (54\%), cytokines $(35 \%)$, bevacizumab $(8 \%)$, and temsirolimus (3\%). Although there was no difference in overall survival (OS) between the two groups, treatment with axitinib resulted in a significantly longer progression-free survival (PFS) compared with sorafenib at 6.7 months vs 4.7 months, respectively $(p<0.0001)$. Common toxicities of axitinib were diarrhea, hypertension, and fatigue. Final survival analysis confirmed no difference in OS, with PFS results continuing to favour axitinib over sorafenib. ${ }^{4}$

\section{Everolimus}

Everolimus is an mTOR inhibitor that binds with high affinity to its intracellular receptor (FKBP12) inhibiting the mTORC1 complex and subsequent cell growth, proliferation, and survival. ${ }^{5}$ Encouraging antitumour activity was demonstrated in the phase 2 trial of everolimus comprising 39 patients with RCC who had progressed on systemic immunotherapy, chemotherapy, and/or TKI therapy, with a PFS greater than six months in approximately $70 \%$ of patients. ${ }^{6}$ In the phase 3 RECORD-1 study, 416 patients from 86 centres who had progressed during or within six months of treatment with sunitinib, sorafenib, or both, were randomly assigned to best supportive care plus either everolimus or placebo. PFS was significantly greater in the everolimus arm (4.9 vs. 
1.9 months; $p<0.001) .{ }^{7}$ Median OS was 14.8 months in the everolimus group and 14.4 months in the placebo group $(p=0.162)$; however, these results were confounded by crossover, as $80 \%$ of patients in the placebo group were switched to open-label everolimus before the final OS analysis. The most common serious adverse events (AEs) with everolimus were infections, dyspnea, and fatigue.

\section{Nivolumab}

Nivolumab is a fully human immunoglobulin G4 PD-1 immune checkpoint inhibitor antibody that inhibits the interaction between PD-1 expressed on activated T-cells and PD-1 ligand (PD-L1) and PD-L2 expressed on tumour cells, with a resulting immunomodulatory and antineoplastic activity. Single-agent nivolumab was compared with everolimus in the randomized, open-label, phase 3 CheckMate-025 trial of 821 patients with advanced clear-cell RCC who had progressed on one or two antiangiogenic therapies. ${ }^{8}$ The primary endpoint was OS and it was significantly greater with nivolumab than with everolimus (25.0 vs. 19.6 months; $\mathrm{p}=0.002$ ). Improved objective response rate (ORR) was noted with nivolumab at $25 \%$ vs. $5 \%(p<0.001)$ along with a better toxicity profile (Grade 3 or 4 event rate of $19 \%$ vs. $37 \%)$. Although, there was no difference in PFS between the two groups, an ad hoc analysis of PFS in patients without progression/death at six months was completed to explore the delayed benefit of nivolumab. Median PFS from this analysis was 15.6 months for nivolumab vs. 11.7 months for everolimus, suggesting improved benefit in responders to therapy from both groups. In general, nivolumab was felt to be quite well-tolerated, with the most common AEs being nausea and pruritus.

\section{Cabozantinib}

Cabozantinib is a TKI with multiple targets, including VEGFR, MET, and AXL. ${ }^{9}$ Upregulation of MET and AXL is noted with sunitinib resistance, making cabozantinib an ideal compound to test in the post-VEGFR-TKI setting. ${ }^{10}$ Cabozantinib was recently tested and approved in the U.S. and Europe in patients with mRCC who had received prior antiangiogenic therapy. The approval was based on the randomized, open-label, phase 3 METEOR trial in which 658 patients with advanced RCC who had undergone prior treatment with one or more anti-VEGFR-TKIs were treated with either cabozantinib or everolimus. ${ }^{11}$ Median PFS among the first 375 randomized subjects was 7.4 months in the cabozantinib-treated group and 3.8 months in the everolimus-treated group $(\mathrm{p}<0.0001)$. In the final analysis, after a median followup of 18.7 months, OS was significantly longer with cabozantinib than with everolimus (21.4 vs. 16.5 months; $p=0.00026) .{ }^{12}$ Cabozantinib was also associated with an improved ORR compared with everolimus $(17 \%$ vs. $3 \%$; $p<0.0001$ ). Although effective, $68 \%$ of patients had Grade 3 or $4 \mathrm{AEs}$ - the most common being hypertension, diarrhea, fatigue, hand-foot syndrome, anemia, hyperglycemia, and hypomagnesemia.

\section{Combination lenvatinib and everolimus}

Lenvatinib is a multireceptor TKI with activity against VEGFR and FGFR. In the U.S., the Food and Drug Administration (FDA) recently approved the combination of lenvatinib and everolimus based on the results of a randomized, phase 2, multicentre study of 153 patients with advanced or mRCC who had progressed on a VEGFR-targeted therapy. ${ }^{13}$ Patients were randomly assigned 1:1:1 to everolimus, lenvatinib, or a combination of lenvatinib plus everolimus. The median PFS was significantly greater for the combination of lenvatinib plus everolimus compared with everolimus alone (14.6 vs. 5.5 months; $\mathrm{p}=0.0005)$, but not compared with lenvatinib alone ( 7.4 months; $p=0.12$ for the comparison). In the post-hoc updated analysis, the median OS was significantly higher among those treated with lenvatinib plus everolimus than among those treated with single-agent everolimus (25.5 months vs. 5.4 months; $p=0.024) .{ }^{13}$ Although impressive results, the combination was found to have a Grade $3 / 4$ toxicity rate of $71 \%$, with the most common toxicities being diarrhea, hypertension, fatigue, anemia, hypertriglyceridemia, nausea, vomiting, and anorexia. Cabozantinib is not yet available in Canada.

\section{Third-line treatment}

To date, Health Canada has not yet approved any agent specifically in the third-line setting. With disease progression following second-line treatment, enrollment into a clinical trial is recommended where possible. ${ }^{1,14}$ There is minimal high-quality evidence to guide choice of therapy in this setting, as the majority of clinical trials have been conducted in the first- or second-line setting.

One prospective study in the third-line setting is the open-label, phase 3 GOLD study comparing dovitinib to sorafenib. Despite positive phase 2 data with this drug, ${ }^{15}$ the GOLD study failed to show any improvement in outcomes with dovitinib. ${ }^{16}$

Other data from phase 3 trials suggest the potential use of approved second-line agents in the third-line setting. In the RECORD-1 trial, $26 \%$ of patients had undergone treatment with two prior anti-VEGFR-TKIs and demonstrated a similar PFS benefit with everolimus to the overall population. ${ }^{7}$ In the METEOR trial, 29\% of patients had undergone prior treatment with two or more VEGFR-TKIs. ${ }^{12}$ Subgroup analysis of this population suggested improved PFS and a trend toward improved OS with cabozantinib use. In the CheckMate-025 
trial, a trend towards improved OS was noted in the $28 \%$ of enrolled patients who had previously been treated with two antiangiogenic regimens. ${ }^{8}$

\section{Conclusion}

The optimal sequencing of targeted therapies for the treatment of mRCC beyond first-line has evolved as new therapies have developed. Cabozantinib (in the U.S. and Europe) and nivolumab have supplanted everolimus as standards of care in the second-line setting. Axitinib remains a secondline option, but it will likely be relegated to the third-line or beyond given its neutral comparison to everolimus, as well as the superiority of cabozantinib and nivolumab to the same. Given the lack of direct comparison though, axitinib will likely be the drug of choice over everolimus. The combination of lenvatinib and everolimus remains promising, but economic factors and a lack of phase 3 data will likely limit accessibility in the Canadian setting. Clinical trials to compare these treatment options to better assess their efficacy would be ideal in clarifying their sequencing. However, this is unlikely to occur, given the movement of many of these options into the first-line setting, novel drug development, and the increasing demand for patients for clinical trials. As such, the ongoing development of predictive biomarkers and real-world data collection and analysis will be of major importance in helping choose the appropriate therapy for patients with mRCC.

At present, available consensus statements and guidelines are helpful in guiding treatment choices. The most recently published European guidelines provide some recommendations on the sequencing of agents in mRCC patients ${ }^{14}$ and the development of a new Canadian consensus statement is underway. Ultimately, the selection of treatments in the second-line setting and beyond will be driven by best evidence, clinical judgement (including consideration of drug toxicity, patient goals, performance status, comorbidities, tolerability of previous treatments), and, of course, drug availability.

\section{References}

1. North SA; Canadian Kidney Cancer Forum 2015. Management of advanced kidney cancer: Canadian Kidney Cancer Forum consensus update. Can Urol Assoc J 2015;9:164-70. hitps://doi.org/10.5489/cuai.2894

2. Rini $B I$, Wilding $G$, Hudes $G$, et al. Phase 2 study of axitinib in sorafenib-refractory metastatic renal cell carcinoma. J Clin Oncol 2009;27:4462-8. https://doi.org/10.1200/JC0.2008.21.7034

3. Rini Bl, Escudier B, Tomczak $P$, et al. Comparative effectiveness of axitinib vs. sorafenib in advanced renal cell carcinoma (AXIS): A randomized, phase 3 trial. Lancet 2011;378:1931-9. https://doi. org/10.1016/S0140-6736(11)61613-9

4. Motzer RJ, Escudier B, Tomczak P, et al. Axitinib vs. sorafenib as second-line treatment for advanced renal cell carcinoma: Overall survival analysis and updated results from a randomized, phase 3 trial. Lancet Oncol 2013;14:552-62. https://doi.org/10.1016/S1470-2045(13)70093-7

5. Anandappa G, Hollingdale A, Eisen T. Everolimus - a new approach in the treatment of renal cell carcinoma. Cancer Manag Res 2010;2:61-70.

6. Amato RJ, Jac J, Giessinger $S$, et al. A phase 2 study with a daily regimen of the oral mTOR inhibitor RADO01 (everolimus) in patients with metastatic, clear-cell renal cell cancer. Cancer 2009;115:2438-46. https://doi.org/10.1002/cncr.24280

7. Motzer RJ, Escudier B, Oudard S, et al; RECORD-1 Study Group. Phase 3 trial of everolimus for metastatic renal cell carcinoma: Final results and analysis of prognostic factors. Cancer 2010; 116:4256-65. https://doi.org/10.1002/cncr.25219

8. Motzer RJ, Escudier B, McDermott DF, et al; CheckMate-025 Investigators. Nivolumab vs. everolimus in advanced renal cell carcinoma. N Engl J Med 2015;373:1803-13. https://doi.org/10.1056/ NEJMoa1510665

9. Yakes FM, Chen J, Tan J, et al. Cabozantinib (XL184), a novel MET and VEGFR2 inhibitor, simultaneously suppresses metastasis, angiogenesis, and tumour growth. Mol Cancer Ther 2011;10:2298-308. https://doi.org/10.1158/1535-7163.MCT-11-0264

10. Grassi P, Verzoni E, Ratta R, et al. Cabozantinib in the treatment of advanced renal cell carcinoma: Design, development, and potential place in the therapy. Drug Des Devel Ther 2016;10:2167-72. https://doi. org/10.2147/DDDT.S104225

11. Choueiri TK, Escudier B, Powles T, et al; METEOR Investigators. Cabozantinib vs. everolimus in advanced renal cell carcinoma. N Engl J Med 2015;373:1814-23. https://doi.org/10.1056/NEJMoal510016

12. Choueiri TK, Escudier B, Powles T, et al; METEOR investigators. Cabozantinib vs. everolimus in advanced renal cell carcinoma (METEOR): Final results from a randomized, open-label, phase 3 trial. Lancet Oncol 2016;17:917-27. https://doi.org/10.1016/S1470-2045(16)30107-3

13. Motzer RJ, Hutson TE, Glen H, et al. Lenvatinib, everolimus, and the combination in patients with metastatic renal cell carcinoma: A randomized, phase 2, open-label, multicentre trial. Lancet Oncol 2015;16:1473-82. https://doi.org/10.1016/S1470-2045(15)00290-9

14. Escudier B, Porta C, Schmidinger M, et al; ESMO Guidelines Committee. Renal cell carcinoma: ESMO clinical practice guidelines for diagnosis, treatment, and followup. Ann Oncol 2016;27 (suppl 5):v58-68.

15. Escudier B, Grünwald V, Ravaud A, et al. Phase 2 results of dovitinib (TKI258) in patients with metastatic renal cell cancer. Clin Cancer Res 2014;20:3012-22. https://doi.org/10.1158/1078-0432. CCR-13-3006

16. Motzer RJ, Porta C, Vogelzang NJ, et al. Dovitinib vs. sorafenib for third-line targeted treatment of patients with metastatic renal cell carcinoma: An open-label, randomized, phase 3 trial. Lancet Oncol 2014;15:286-96. https://doi.org/10.1016/S1470-2045(14)70030-0 